\title{
Reversible Quantum Information Spreading in Many-Body Systems near Criticality
}

\author{
Quirin Hummel, Benjamin Geiger®," Juan Diego Urbina, and Klaus Richter \\ Institut für Theoretische Physik, Universität Regensburg, D-93040 Regensburg, Germany
}

(Received 22 December 2018; published 15 October 2019)

\begin{abstract}
Quantum chaotic interacting $N$-particle systems are assumed to show fast and irreversible spreading of quantum information on short (Ehrenfest) time scales $\sim \log N$. Here, we show that, near criticality, certain many-body systems exhibit fast initial scrambling, followed subsequently by oscillatory behavior between reentrant localization and delocalization of information in Hilbert space. We consider both integrable and nonintegrable quantum critical bosonic systems with attractive contact interaction that exhibit locally unstable dynamics in the corresponding many-body phase space of the large- $N$ limit. Semiclassical quantization of the latter accounts for many-body correlations in excellent agreement with simulations. Most notably, it predicts an asymptotically constant local level spacing $\hbar / \tau$, again given by $\tau \sim \log N$. This unique timescale governs the long-time behavior of out-of-time-order correlators that feature quasiperiodic recurrences indicating reversibility.
\end{abstract}

DOI: 10.1103/PhysRevLett.123.160401

The dynamics of quantum information in complex many-body (MB) systems presently attracts a lot of attention $[1,2]$ ranging from atomic and condensed quantum matter to high energy physics. The evolution of an (excited) quantum MB system towards a state of thermal equilibrium usually goes along with the scrambling of quantum correlations, encoded in the initial state, across the system's many degrees of freedom (d.o.f.). Such dynamics requires an improved understanding of MB quantum chaos and the link with thermalization [3-6] and its suppression $[1,7,8]$.

Echo protocols, measuring how a perturbation affects successive forward and backward propagations in time, sensitively probe the stability of complex quantum dynamics. Here, out-of-time-order correlators (OTOCs) [9-11]

$$
C(t)=\left\langle[\hat{W}(t), \hat{V}]^{\dagger}[\hat{W}(t), \hat{V}]\right\rangle
$$

play a central role, with first experimental implementations [12-14], allowing us to distinguish various classes of $\mathrm{MB}$ systems by their operator growth. On the one side, there are slow scramblers, such as systems in the MB localized phase exhibiting logarithmically slow operator spreading [15-18] or, e.g., Luttinger liquids [19] showing only quadratic increase. On the other side, an exponentially fast initial growth of OTOCs is commonly viewed as a quantum signature of $\mathrm{MB}$ chaos. Examples comprise systems with holographic duals to black holes $[10,20]$, the Sachdev-YeKitaev model [11,21-23], and condensed matter systems close to a quantum phase transition (QPT) [24-27] or exhibiting chaos in the classical limit of large particle number $N$. In such large- $N$ systems, the exponential growth rate for OTOCs is given by the Lyapunov exponent of their classical counterpart $[10,27-34]$ and prevails up to the
Ehrenfest $\log N$ time where MB quantum interference sets in $[32,35]$. Subsequent OTOC time evolution toward an ergodic limit is then often governed by slow classical modes [36].

Here, we show that exponentially fast scrambling need not necessarily lead to quantum information loss: There exist systems exhibiting initial growth of complexity without saturation; i.e., after a quench to an interacting system close to criticality, the OTOCs do not show monotonic saturation; instead, the correlations imprinted initially can be periodically retrieved.

Quantum critical large- $N$ systems are particularly suited for considering the inter-relation between the spreading of correlations, quantified through OTOCs, and corresponding nonlinear classical mean-field (MF) dynamics. There, critical phenomena are often viewed as quantum manifestations of structural changes in classical phase space, associated with unstable MF motion close to separatrices. While corresponding studies [37-43] commonly invoke a classical MF analysis, we will show that MB semiclassical quantization beyond MF allows for a precise characterization of the locally unstable quantum dynamics. Therefore, in the language of renormalization group analysis, we expect our results to be valid for any dimension within lower and upper critical dimension as long as a MF (classical) limit exists [44].

While in generic quantum chaotic systems the Ehrenfest time $\sim \log N$ is distinctly shorter than the Heisenberg time (associated with the inverse mean level spacing), we will show that these two scales, indeed, coincide for certain quantum critical systems where an adiabatic separation allows for an effective 1D description. Quantization of their locally hyperbolic MB dynamics implies two inter-related features: Even though the dynamics may be separable, 
OTOCs still grow exponentially with a rate given by the local MF instability exponent $\lambda_{s}$ up to times $\left(1 / \lambda_{s}\right) \log N$. Second, the inverse mean level spacing in the relevant spectral region also scales as $\log N$. Hence, the quantum critical dynamics is governed by $\log N$ as the sole time scale.

Remarkably, this level spacing turns out to be asymptotically constant, approaching a harmonic oscillator spectrum, although the underlying hyperbolic dynamics is unstable and rather corresponds to an inverted oscillator [46]. This equidistant level spacing implies strong, periodic quantum recurrences on short $\log N$ scales that dominate OTOCs and, hence, reflect unscrambling of information in quantum critical MB systems. After showing this behavior in a prototypical integrable model, we consider a nonintegrable extension and confirm the robustness of this feature, indicating that it is not linked to integrability but is characteristic for QPTs driven dominantly by a single d.o.f. On the contrary, in generic chaotic MB systems, randomlike evolution is expected for enormously long (Heisenberg) times beyond which the spectral discreteness eventually enforces recurrences [48].

Quantum critical atomic Bose gas.-As a generic example of critical behavior, we consider the 1D attractive Bose gas with periodic boundary conditions (attractive Lieb-Liniger model) [50-52]. While its Hamiltonian

$\hat{\mathcal{H}}=\int_{0}^{2 \pi} d \theta \hat{\Psi}^{\dagger}(\theta)\left(-\partial_{\theta}^{2}\right) \hat{\Psi}(\theta)-\frac{\pi \tilde{\alpha}}{2}\left[\hat{\Psi}^{\dagger}(\theta)\right]^{2}[\hat{\Psi}(\theta)]^{2}$,

with bosonic field operators $\hat{\Psi}$ and $\hat{\Psi}^{\dagger}$, describes quasi-1D ultracold atomic gases with interactions parametrized by $\tilde{\alpha}$ [53-55], its MF dynamics is governed by the GrossPitaevskii equation. It exhibits a QPT at a critical coupling $\tilde{\alpha} N=1$ [51,56,57], where the homogeneous condensate starts forming a bright soliton. Although for finite $N$, eigenvalues of the quantum integrable Hamiltonian (2) can be, in principle, found through Bethe ansatz $[52,58]$, this does not allow for systematically treating the $N \rightarrow \infty$ limit, except for special states $[59,60]$. Instead, first, we truncate [61] $\hat{\Psi}(\theta)$ to the lowest three momentum modes,

$$
\hat{\Psi}(\theta)=\left(\hat{a}_{0}+\hat{a}_{-1} e^{-\mathrm{i} \theta}+\hat{a}_{1} e^{\mathrm{i} \theta}\right) / \sqrt{2 \pi},
$$

as commonly done for exact diagonalization [56,71,72]—a good approximation for $\tilde{\alpha} N<1$ that also contains all the physics relevant for understanding the QPT and its precursors for $\tilde{\alpha} N \geq 1[51,52,57,71]$. The generalization to the nonintegrable five-mode model will be discussed later. The model (2), (3) near the QPT mimics black holes as graviton condensates [71-73], can be essentially realized using ultracold spin-1 atoms [74] and has attracted considerable attention [75-77] for time crystals [78].

Classical MF limit.-Besides the energy, the particle number and total (angular) momentum are also conserved:

$$
\hat{N}=\sum_{k} \hat{a}_{k}^{\dagger} \hat{a}_{k}, \quad \hat{K}=\sum_{k} k \hat{a}_{k}^{\dagger} \hat{a}_{k} .
$$

Hence, the truncation to three modes, in contrast to five or more modes [79], renders the system integrable in that its large- $N$ MF limit, formally representing a classical limit, is integrable. This allows for devising a MB version of semiclassical torus quantization $[80,81]$ to analytically find the spectrum and wave functions being asymptotically exact for $N \rightarrow \infty$. To this end, we write the operators in symmetric order and replace $\hat{a}_{k} \mapsto \sqrt{n_{k}} e^{\mathrm{i} \vartheta_{k}}$ for $k=-1,0$, 1 , where $\left(n_{k}, \vartheta_{k}\right)$ are continuous classical conjugate variables. Using

$$
\tilde{N} \equiv n_{-1}+n_{0}+n_{1}, \quad K \equiv n_{1}-n_{-1},
$$

and considering $K=0$, the classical energy per particle is, defining $\alpha=\tilde{\alpha} \tilde{N}$, [61]

$$
\frac{E}{\tilde{N}}=\omega(z, \varphi)+\alpha\left(-\frac{1}{4}+\frac{3}{2 \tilde{N}}-\frac{9}{8 \tilde{N}^{2}}\right)-\frac{1}{\tilde{N}},
$$

where the classical dynamics is completely determined by

$$
\omega(z, \varphi)=(1-z)\left\{1-\alpha\left[(1-z) / 8+z \cos ^{2} \varphi\right]\right\},
$$

with phase space coordinates

$$
z=\frac{n_{0}}{\tilde{N}}, \quad \varphi=\vartheta_{0}-\frac{1}{2}\left(\vartheta_{1}+\vartheta_{-1}\right), \quad\{z, \varphi\}=\frac{1}{\tilde{N}} .
$$

Note that $1 / \tilde{N}$ only enters as an effective quantum of action in the Poisson brackets [82].

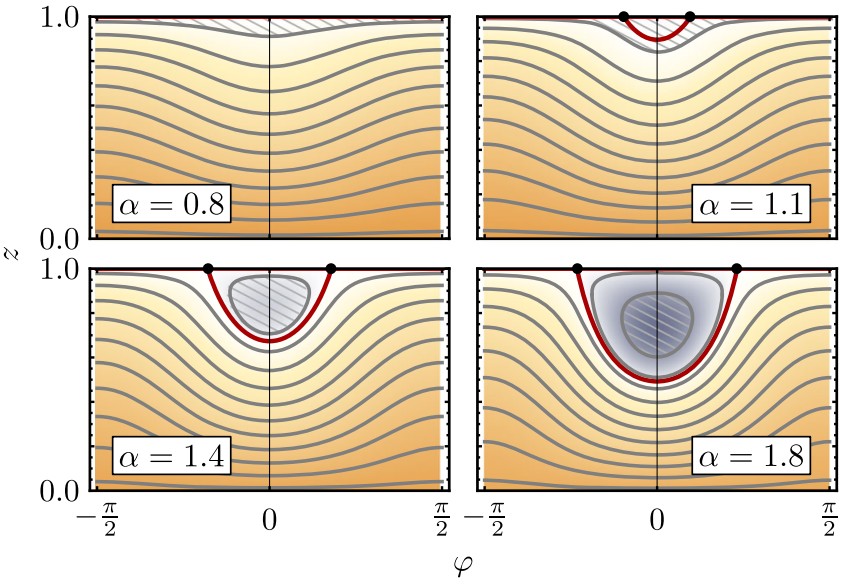

FIG. 1. Phase space portrait of energy $\omega(z, \varphi)$, Eq. (7), of the classical dynamics for the three-mode model of attractive bosons in $1 \mathrm{D}$, for different values of interaction $\alpha$. Here, $z$ denotes the relative occupation of the noninteracting ground state and $\varphi$ the conjugate angle. For $\alpha>1$, a global energy minimum (cross) and a separatrix (red) connecting two hyperbolic fixed points (dots at $z=1$ ) appear. Gray lines represent the orbits (tori) that fulfill the quantization condition (10) for $N=20$. Note that the quantized orbits change their character sequentially. 
The Hamiltonian $\omega(z, \varphi)$ involves different types of classical trajectories following lines of constant energy in phase space with periodicity $\varphi \mapsto \varphi+\pi$, see Fig. 1. For $\alpha<1$ all trajectories are deformed horizontal lines (rotations). For $\alpha>1$ an island centered around a new minimum energy fixed point emerges with orbits vibrating in $\varphi$, similar as for the pendulum. This goes along with the formation of a separatrix at $E=E_{\text {sep }}(\omega=0)$ associated with two hyperbolic fixed points at $z=1$ and characterized by (in)stability exponents

$$
\lambda_{s}^{(1,2)}=2 \sqrt{\alpha-1} \equiv \lambda_{s} .
$$

Semiclassical quantization.-To study genuine quantum effects, we go beyond the classical MF picture using semiclassical torus quantization. While related WKB approaches, devised for one-dimensional systems, were successfully used in two-site models [83-85], we adapt a multidimensional generalization. In the MB context, it yields the quantization rules

$\frac{1}{2 \pi} \oint d \varphi[1-z(\omega, \varphi)]=\frac{m+\frac{1}{2}}{\tilde{N}}$,

$m=0,1, \ldots,\lfloor N / 2\rfloor, \quad \tilde{N}=N+3 / 2, \quad N=0,1, \ldots$

Equations (10) effectively quantize the phase space areas bounded by the lines $\omega=$ const (shaded areas in Fig. 1 for $m=0$ ), giving rise to energies $\omega_{m}$ and $E_{m}$, in perfect agreement with results from exact diagonalization, see Fig. 2.

Quantum phase transition.-The QPT for $N \rightarrow \infty$ is associated with the ground state corresponding to the quantized orbit enclosing the phase space area $1 /(2 \tilde{N}) \rightarrow$ 0 that is always vibrational for $\alpha>1$ if $\tilde{N}$ is large enough. Its energy scales as $\omega_{\min } \sim-(\alpha-1)^{2}$, in contrast to

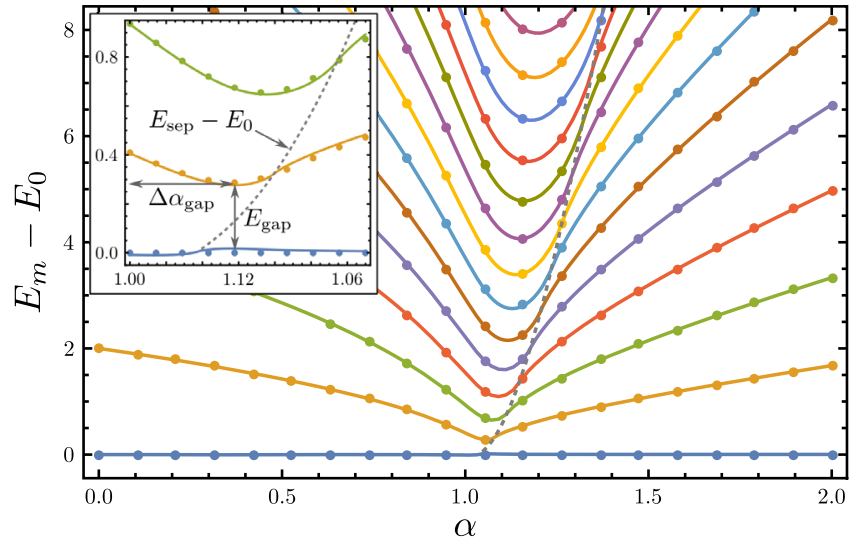

FIG. 2. Excitation spectrum $E_{m}-E_{0}$ for $N=300$ and $0 \leq \alpha \leq$ 2 within three-mode approximation, Eq. (3). Symbols and lines denote numerical and semiclassical results, respectively. The gray dotted line indicates inflection points, the finite size precursor of an excited state QPT, where quantized orbits cross the separatrix (in Fig. 1). Inset: closeup around $\alpha=1$. $\omega_{\min }=0$ for $\alpha<1$ where the quantized orbit approaches $z=1$, leading to the nonanalytic dependence on $\alpha$ of the MF ground state at $\alpha=1$. Precursors of such nonanalyticity for finite $\tilde{N}$ appear for every quantized orbit changing from rotation to vibration upon tuning $\alpha$. This is reflected in the sequence of avoided crossings in Fig. 2 building up an excited state QPT when $\tilde{N} \rightarrow \infty[38,41]$. Remarkably, Eq. (10) even allows us [86] to analytically obtain the scaling laws [87] governing the approach of $\Delta \alpha_{\text {gap }} \sim N^{-2 / 3}$ and $E_{\text {gap }} \sim N^{-1 / 3}$ (see inset of Fig. 2) to their MF values, in perfect agreement with numerical and heuristic observations $[51,56,72]$.

Hence, MB semiclassical quantization goes beyond the Bogoliubov picture of $[51,57]$ where the excitation spectrum collapses to zero at the MF critical coupling $\alpha=1$. Instead, for finite $\tilde{N}$ we find huge accumulations of levels around the separatrix. This precursor of an excited state QPT leads to characteristic features in the spectra and to the emergence of a local log time scale. An asymptotic $N \gg 1$ analysis [61] of (10) that generally holds close to a separatrix [61] yields the average density of states

$$
\bar{\varrho}(E)=\frac{-1}{2 \pi \lambda} \log \left(\frac{\left|E-E_{\mathrm{sep}}\right|}{\tilde{N}}\right)+\mathcal{O}(1),
$$

with a characteristic logarithmic divergence at $E=E_{\text {sep }}$ (see Fig. 3). Here, $\lambda=\lambda_{s} / 2=\sqrt{\alpha-1}$, Eq. (9), and the $\mathcal{O}(1)$ finite size correction involves a system specific timescale related to the traversal along the separatrix [61].

Asymptotically constant level spacing and log time.Most notably, evaluating (10) close to $E_{\text {sep }}$, one finds [61] that a set of levels, growing in number logarithmically with $N$, becomes asymptotically equidistant with level spacing $\Delta E$, see Fig. 4. The associated time scale [61]
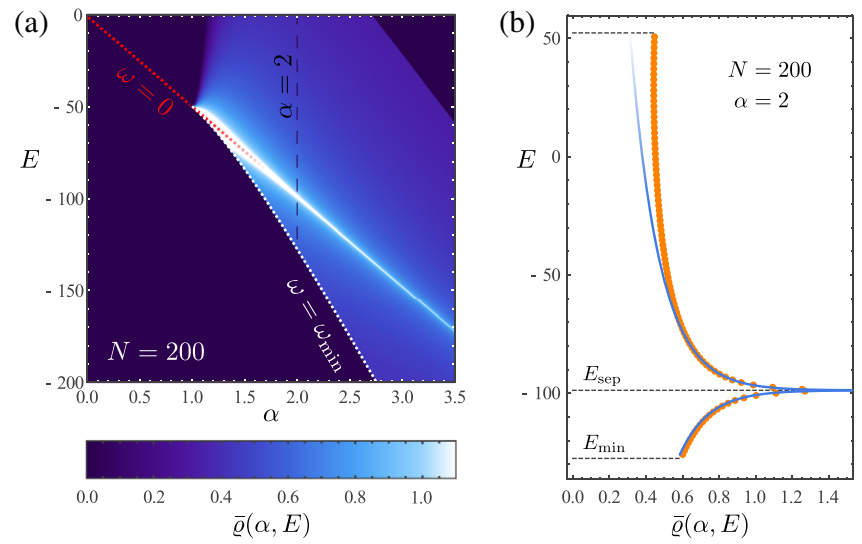

FIG. 3. (a) Asymptotic $N \gg 1$ density of states $\bar{\rho}(\alpha, E)$, Eq. (11), showing level bunching (bright straight line) around the precursor of the excited state QPT at $E=E_{\text {sep }}$ in the supercritical regime $\alpha>1$. (b) Slice of $\bar{\rho}(\alpha, E)$ at $\alpha=2$ compared with numerically calculated inverse level gaps (orange symbols) exhibiting a characteristic logarithmic divergence. 


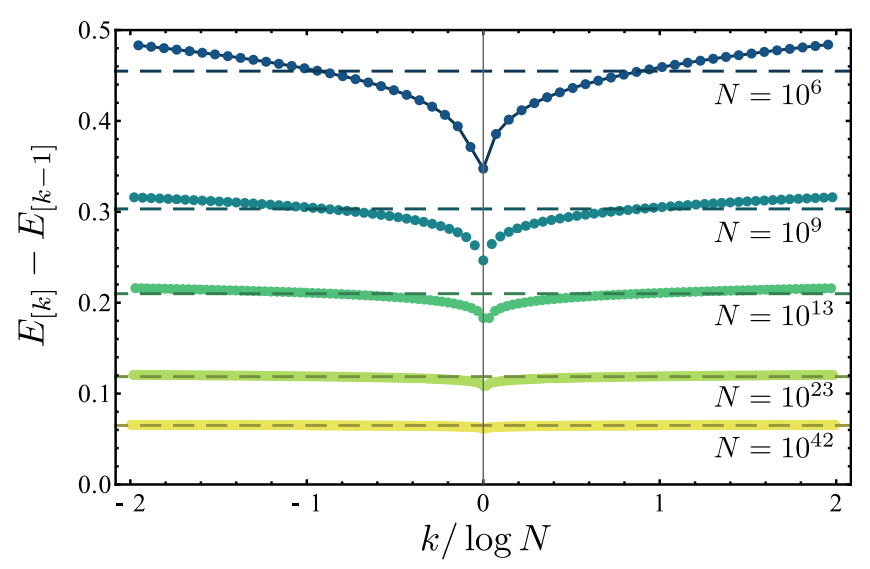

FIG. 4. Asymptotic level spacings $E_{[k]}-E_{[k-1]}$ (symbols) obtained from (11) [61], where $E_{[k=0]}$ refers to the level closest to the separatrix energy (excited state QPT), approaching the characteristic constant spacing $\Delta E$, Eq. (12), (dashed line) for $\alpha=2$ and $N=10^{6}, 10^{9}, 10^{13}, 10^{23}, 10^{42}$ (the latter with relevance for black hole physics) from top (blue) to bottom (yellow) within windows with $\sim \log N$ levels. Energies obtained from full semiclassical quantization, (10), (solid line) for $N=10^{6}$ confirm the validity of Eq. (11).

$$
\tau=\frac{2 \pi}{\Delta E}=\frac{1}{\lambda} \log \tilde{N}+\mathcal{O}(1)
$$

is the Heisenberg time corresponding to the local spectral gap $\Delta E$, but exhibits a striking similarity with the Ehrenfest time $\tau_{E}=\left(1 / \lambda_{L}\right) \log \hbar_{\text {eff }}^{-1}$ with $\hbar_{\text {eff }}=\hbar$ and $1 / \tilde{N}$ in chaotic single particle [88], respectively, MB systems [32] with Lyapunov exponent $\lambda_{L}$. This justifies to relate (12) to a local Ehrenfest time associated with the dynamical instability characteristic of critical behavior. Because of the universality of (11) and (12), supported by the classical renormalization group analysis of [89], this turns out to be a generic behavior close to hyperbolic fixed points. The crossover of the Heisenberg time from usually algebraic in $N$ to log time behavior is not shared by generic chaotic systems.

Out-of-time-order correlator-We address the drastic consequences of this transition for the evolution of quantum MB correlations: We quantify the spreading of information through the OTOC, Eq. (1),

$$
C(t)=-N^{-4}\left\langle\psi\left|\left[\hat{n}_{0}(0), \hat{n}_{0}(t)\right]^{2}\right| \psi\right\rangle,
$$

for operators $\hat{n}_{0}(t) / N$. In chaotic systems quasiclassical arguments $[9,10,28]$ confirmed by MB semiclassical theory [32] predict a short-time behavior $C(t) \sim \hbar^{2} e^{2 \lambda_{L} t}$ passing into a saturation regime at $\tau_{E}$. Although the system (3) is integrable, we can use similar arguments to predict that in the quantum critical regime the dynamical instability close to the hyperbolic fixed points also causes such an exponential behavior, (a)
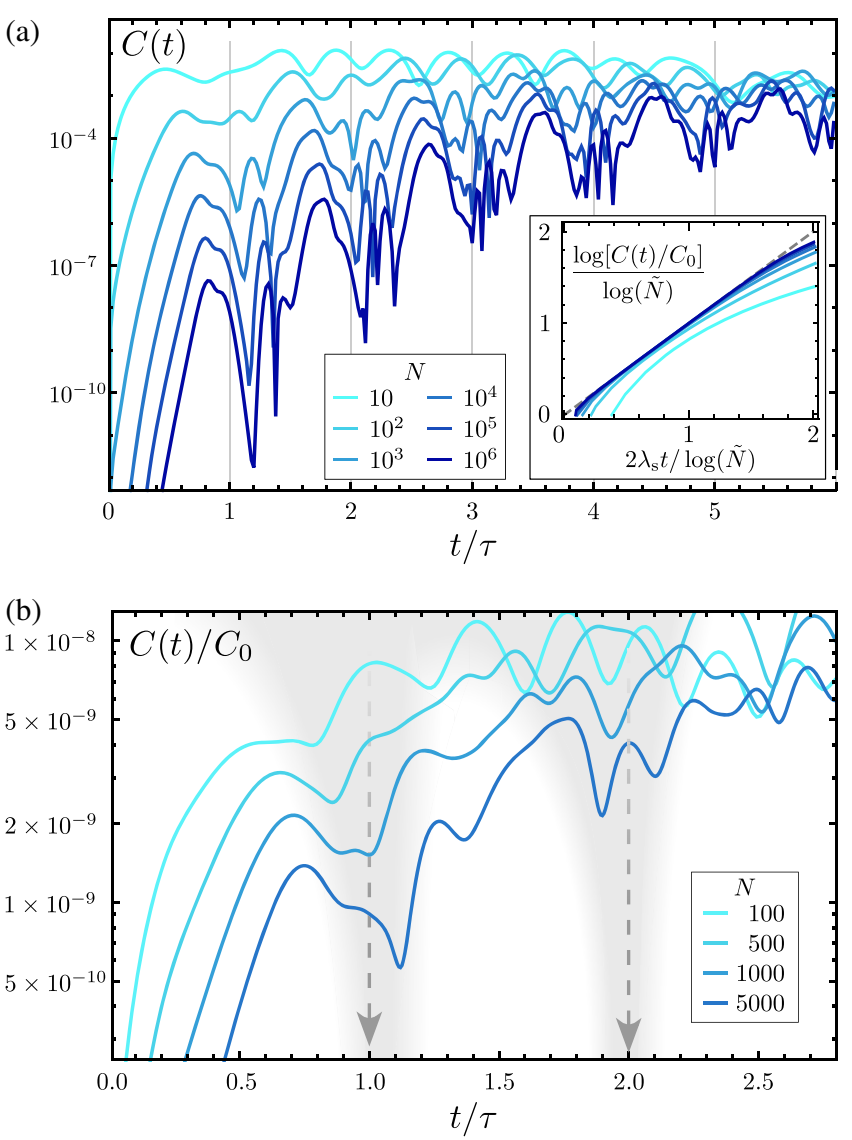

FIG. 5. Oscillatory time evolution of the OTOC (13) after a quench of the noninteracting condensate across the mean-field critical point, reflecting scrambling and unscrambling (i.e., indicating reversibility) close to criticality for various particle numbers $N$. (a) $C(t)$ exhibits distinct, approximately $\tau$-periodic oscillations where $\tau$, Eq. (12), is the local Ehrenfest time [threemode model (3) with $\alpha=2]$. Inset: Initial growth of $C(t)$ approaching the exponent $2 \lambda_{s} t$ (dashed line) with increasing $N$, thereby confirming Eq. (14). (b) Tendency towards periodicity (suggested in gray) for the nonintegrable extended five-mode model $(|k| \leq 2$, see [61]) for $\alpha=1.05$, with a period of the form (12) with $\lambda \approx 0.21$, consistently found in the level spacings and the classical instability. The prefactors $C_{0}$ facilitate the comparison.

$$
C(t) \sim\left(\hbar_{\mathrm{eff}}^{2} / \tilde{N}^{2}\right) e^{2 \lambda_{s} t},
$$

but with a rate given by the (in)stability exponents $\lambda_{s}$, Eq. (9) (see [43] for a related result for chains of large spins).

Within the present MB model we have numerical access to huge particle numbers and, hence, can thoroughly check the commonly assumed exponential growth of OTOCs in the truly semiclassical large- $N$ limit, as well as associated log-time effects. In Fig. 5, we present numerical results for $C(t)$ computed from (13) after imposing an interaction quench to the noninteracting ground state $|\psi\rangle=$ $1 / \sqrt{N !}\left(\hat{a}_{0}^{\dagger}\right)^{N}|0\rangle$. The inset displays the short time behavior 
of $\log C(t)$ up to the Ehrenfest time scale $\tau_{E}=\left(1 / \lambda_{s}\right) \log \tilde{N}$ for $N=10^{2}$ to $10^{6}$, showing convergence to the slope $2 \lambda_{s} t$ (dashed line) predicted in (14).

Unscrambling of correlations.-Figure 5(a) shows that instead of monotonically approaching a plateau for $t>\tau_{E}$, as in generic chaotic MB systems, $C(t)$ exhibits distinct oscillations with a period given by the $\log$ time $\tau$, (12), that includes finite-size corrections [61] and, hence, slightly differs from $\tau_{E}$. Since $C(t)$ is a measure of information spreading, these oscillations reflect reversibility of quantum information flux in Hilbert space as a result of genuine MB interference. This is supplemented by corresponding oscillations in the evolution of entanglement encoded in the one-body entropy [61]. Close to criticality, $C(t)$ is dominated by an increasing (with $\log N$ ) number of states close to $E_{\text {sep }}$ [61] where the spectrum gets asymptotically equidistant (Fig. 4). This induces revivals (getting more and more pronounced with increasing $N$ ) associated with a unique time scale, the log time $\tau,(12)$, taking the role of a Heisenberg time close to criticality. To clear-cut show this asymptotic periodicity, here deduced from MB separatrix quantization, requires large- $N$ regimes that, to the best of our knowledge, are not accessible with present numerical methods for chaotic MB systems.

To assess the latter and to explore the generality of our findings, we further relax the truncation (3) to five modes $(|k| \leq 2)$, implying unstable nonintegrable dynamics. Within the bounds of numerical tractability $\left[N=\mathcal{O}\left(10^{4}\right)\right]$, we verify that this quantum critical MB system, despite being nonintegrable, exhibits the same tendency towards periodic revivals, see Fig. 5(b). Via adiabatic separation, we can again attribute this behavior to locally hyperbolic MF dynamics of a single dominant d.o.f. [61]. Moreover, we find the same interrelation between the MF instability $\lambda_{s}$, the period $\tau$, and the local level spacing as in the integrable three-mode model.

In conclusion, by means of many-body semiclassical quantization, we could explain the exponentially fast scrambling and buildup of correlations in quantum systems that are critical, but not globally chaotic. We uncovered a generic mechanism for fast dynamics near a quantum critical point. Moreover, for large $N$, we demonstrated the emergence of nearly equidistant spectra giving rise to recurrences in OTOCs on $\log N$ time scales, resembling features of time crystals [78]. Their observation should be in experimental reach since, e.g., recurrences based on stable dynamics have already been observed in a system with thousands of atoms [90]. Our analysis of the nonintegrable five-mode model shows that such memory effects do not require integrability and indicates their existence in larger classes of critical systems with a dynamical decoupling of a dominant unstable mode from other collective degrees of freedom. Moreover, our results shed light on generic mechanisms governing the dynamics at (excited-state) quantum phase transitions beyond mean field.
We acknowledge funding through the Studienstiftung des Deutschen Volkes (B.G.) and the Deutsche Forschungsgemeinschaft through Project No. Ri681/14-1.

* Corresponding author. benjamin.geiger@ur.de

[1] E. Altman, Many-body localization and quantum thermalization, Nat. Phys. 14, 979 (2018).

[2] B. Swingle, Unscrambling the physics of out-of-time-order correlators, Nat. Phys. 14, 988 (2018).

[3] M. Srednicki, Chaos and quantum thermalization, Phys. Rev. E 50, 888 (1994).

[4] M. Rigol, V. Dunjko, and M. Olshanii, Thermalization and its mechanism for generic isolated quantum systems, Nature (London) 452, 854 (2008).

[5] J. Eisert, M. Friesdorf, and C. Gogolin, Quantum manybody systems out of equilibrium, Nat. Phys. 11, 124 (2015).

[6] A. M. Kaufman, M. E. Tai, A. Lukin, M. Rispoli, R. Schittko, P. M. Preiss, and M. Greiner, Quantum thermalization through entanglement in an isolated many-body system, Science 353, 794 (2016).

[7] R. Nandkishore and D. A. Huse, Many-body localization and thermalization in quantum statistical mechanics, Annu. Rev. Condens. Matter Phys. 6, 15 (2015).

[8] M. Schreiber, S. S. Hodgman, P. Bordia, H. P. Lüschen, M. H. Fischer, R. Vosk, E. Altman, U. Schneider, and I. Bloch, Observation of many-body localization of interacting fermions in a quasi-random optical lattice, Science 349, 842 (2015).

[9] A. I. Larkin and Y. N. Ovchinnikov, Quasiclassical method in the theory of superconductivity, Zh. Eksp. Teor. Fiz. 55, 2262 (1969) [Sov. Phys. JETP 28, 1200 (1969)].

[10] J. Maldacena, S. H. Shenker, and D. Stanford, A bound on chaos, J. High Energy Phys. 16 (2016) 106.

[11] J. Maldacena and D. Stanford, Remarks on the Sachdev-YeKitaev model, Phys. Rev. D 94, 106002 (2016).

[12] M. Gärttner, J. G. Bohnet, A. Safavi-Naini, M. L. Wall, J. J. Bollinger, and A.M. Rey, Measuring out-of-time-order correlations and multiple quantum spectra in a trappedion quantum magnet, Nat. Phys. 13, 781 (2017).

[13] J. Li, R. Fan, H. Wang, B. Ye, B. Zeng, H. Zhai, X. Peng, and J. Du, Measuring Out-of-Time-Order Correlators on a Nuclear Magnetic Resonance Quantum Simulator, Phys. Rev. X 7, 031011 (2017).

[14] K. X. Wei, C. Ramanathan, and P. Cappellaro, Exploring Localization in Nuclear Spin Chains, Phys. Rev. Lett. 120, 070501 (2018).

[15] Y. Chen, Universal logarithmic scrambling in many body localization, arXiv:1608.02765.

[16] Y. Huang, Y.-L. Zhang, and X. Chen, Out-of-time-ordered correlators in many-body localized systems, Ann. Phys. (Amsterdam) 529, 1600318 (2017).

[17] R. Fan, P. Zhang, H. Shen, and H. Zhai, Out-of-time-order correlation for many-body localization, Sci. Bull. 62, 707 (2017).

[18] B. Swingle and D. Chowdhury, Slow scrambling in disordered quantum systems, Phys. Rev. B 95, 060201(R) (2017). 
[19] B. Dóra and R. Moessner, Out-of-Time-Ordered Density Correlators in Luttinger Liquids, Phys. Rev. Lett. 119, 026802 (2017).

[20] J. S. Cotler, G. Gur-Ari, M. Hanada, J. Polchinski, P. Saad, S. H. Shenker, D. Stanford, A. Streicher, and M. Tezuka, Black holes and random matrices, J. High Energy Phys. 17 (2017) 118.

[21] A. Kitaev, in Proceedings at KITP, 2015, http://online .kitp.ucsb.edu/online/entangled15/kitaev/, http://online.kitp .ucsb.edu/online/entangled15/kitaev2/.

[22] J. Polchinski and V. Rosenhaus, The spectrum in the Sachdev-Ye-Kitaev model, J. High Energy Phys. 16 (2016) 1.

[23] A. A. Patel and S. Sachdev, Quantum chaos on a critical Fermi surface, Proc. Natl. Acad. Sci. U.S.A. 114, 1844 (2017).

[24] H. Shen, P. Zhang, R. Fan, and H. Zhai, Out-of-time-order correlation at a quantum phase transition, Phys. Rev. B 96, 054503 (2017).

[25] M. Heyl, F. Pollmann, and B. Dóra, Detecting Equilibrium and Dynamical Quantum Phase Transitions in Ising Chains via Out-of-Time-Ordered Correlators, Phys. Rev. Lett. 121, 016801 (2018).

[26] Y. Alavirad and A. Lavasani, Scrambling in the Dicke model, Phys. Rev. A 99, 043602 (2019).

[27] J. Chávez-Carlos, B. López-del-Carpio, M. A. BastarracheaMagnani, P. Stránský, S. Lerma-Hernández, L. F. Santos, and J.G. Hirsch, Quantum and Classical Lyapunov Exponents in Atom-Field Interaction Systems, Phys. Rev. Lett. 122, 024101 (2019).

[28] B. Swingle, G. Bentsen, M. Schleier-Smith, and P. Hayden, Measuring the scrambling of quantum information, Phys. Rev. A 94, 040302(R) (2016).

[29] E. B. Rozenbaum, S. Ganeshan, and V. Galitski, Lyapunov Exponent and Out-of-Time-Ordered Correlator's Growth Rate in a Chaotic System, Phys. Rev. Lett. 118, 086801 (2017).

[30] A. Bohrdt, C. B. Mendl, M. Endres, and M. Knap, Scrambling and thermalization in a diffusive quantum many-body system, New J. Phys. 19, 063001 (2017).

[31] T. Scaffidi and E. Altman, Semiclassical theory of manybody quantum chaos and its bound, arXiv:1711.04768.

[32] J. Rammensee, J. D. Urbina, and K. Richter, ManyBody Quantum Interference and the Saturation of Out-ofTime-Order Correlators, Phys. Rev. Lett. 121, 124101 (2018).

[33] I. García-Mata, M. Saraceno, R. A. Jalabert, A. J. Roncaglia, and D. A. Wisniacki, Chaos Signatures in the Short and Long Time Behavior of the Out-of-Time Ordered Correlator, Phys. Rev. Lett. 121, 210601 (2018).

[34] R. A. Jalabert, I. García-Mata, and D. A. Wisniacki, Semiclassical theory of out-of-time-order correlators for lowdimensional classically chaotic systems, Phys. Rev. E 98, 062218 (2018).

[35] S. Tomsovic, P. Schlagheck, D. Ullmo, J.-D. Urbina, and K. Richter, Post-Ehrenfest many-body quantum interferences in ultracold atoms far out of equilibrium, Phys. Rev. A 97, 061606(R) (2018).

[36] C. W. von Keyserlingk, T. Rakovszky, F. Pollmann, and S. L. Sondhi, Operator Hydrodynamics, OTOCs, and
Entanglement Growth in Systems without Conservation Laws, Phys. Rev. X 8, 021013 (2018).

[37] C. Emary and T. Brandes, Chaos and the quantum phase transition in the Dicke model, Phys. Rev. E 67, 066203 (2003).

[38] M. A. Caprio, P. Cejnar, and F. Iachello, Excited state quantum phase transitions in many-body systems, Ann. Phys. (Amsterdam) 323, 1106 (2008).

[39] V. M. Bastidas, P. Pérez-Fernández, M. Vogl, and T. Brandes, Quantum Criticality and Dynamical Instability in the Kicked-Top Model, Phys. Rev. Lett. 112, 140408 (2014).

[40] P. Stránský, M. Macek, and P. Cejnar, Excited-state quantum phase transitions in systems with two degrees of freedom: Level density, level dynamics, thermal properties, Ann. Phys. (Amsterdam) 345, 73 (2014).

[41] M. A. Bastarrachea-Magnani, S. Lerma-Hernández, and J. G. Hirsch, Thermal and quantum phase transitions in atom-field systems: a microcanonical analysis, J. Stat. Mech. (2016) 093105.

[42] D. Rubeni, J. Links, P. S. Isaac, and A. Foerster, Two-site Bose-Hubbard model with nonlinear tunneling: Classical and quantum analysis, Phys. Rev. A 95, 043607 (2017).

[43] S. Pappalardi, A. Russomanno, B. Žunkovič, F. Iemini, A. Silva, and R. Fazio, Scrambling and entanglement spreading in long-range spin chains, Phys. Rev. B 98, 134303 (2018).

[44] Following [45], criticality occurs above the lower, while quantum fluctuations become subdominant above the upper critical dimension.

[45] P. M. Chaikin and T. C. Lubensky, Principles of Condensed Matter Physics (Cambridge University Press, Cambridge, England, 1995).

[46] See [47] for similar results for the $x p$ model in $\mathrm{AdS}_{2}$.

[47] J. Molina-Vilaplana and G. Sierra, An xp model on $\mathrm{AdS}_{2}$ spacetime, Nucl. Phys. B877, 107 (2013).

[48] Such recurrences are exceptional and exist only at particular wavelengths [49].

[49] S. Tomsovic and J. H. Lefebvre, Can Wave Packet Revivals Occur in Chaotic Quantum Systems? Phys. Rev. Lett. 79, 3629 (1997).

[50] E. H. Lieb and W. Liniger, Exact analysis of an interacting Bose gas. I. The general solution and the ground state, Phys. Rev. 130, 1605 (1963).

[51] R. Kanamoto, H. Saito, and M. Ueda, Quantum phase transition in one-dimensional Bose-Einstein condensates with attractive interactions, Phys. Rev. A 67, 013608 (2003).

[52] A. G. Sykes, P. D. Drummond, and M. J. Davis, Excitation spectrum of bosons in a finite one-dimensional circular waveguide via the Bethe ansatz, Phys. Rev. A 76, 063620 (2007).

[53] K. E. Strecker, G. B. Partridge, A. G. Truscott, and R. G. Hulet, Formation and propagation of matter-wave soliton trains, Nature (London) 417, 150 (2002).

[54] L. Khaykovich, F. Schreck, G. Ferrari, T. Bourdel, J. Cubizolles, L. D. Carr, Y. Castin, and C. Salomon, Formation of a matter-wave bright soliton, Science 296, 1290 (2002).

[55] C. Chin, R. Grimm, P. Julienne, and E. Tiesinga, Feshbach resonances in ultracold gases, Rev. Mod. Phys. 82, 1225 (2010). 
[56] R. Kanamoto, H. Saito, and M. Ueda, Symmetry Breaking and Enhanced Condensate Fraction in a Matter-Wave Bright Soliton, Phys. Rev. Lett. 94, 090404 (2005).

[57] R. Kanamoto, H. Saito, and M. Ueda, Critical fluctuations in a soliton formation of attractive Bose-Einstein condensates, Phys. Rev. A 73, 033611 (2006).

[58] K. Sakmann, A. I. Streltsov, O. E. Alon, and L.S. Cederbaum, Exact ground state of finite Bose-Einstein condensates on a ring, Phys. Rev. A 72, 033613 (2005).

[59] D. Flassig, A. Franca, and A. Pritzel, Large- $N$ ground state of the Lieb-Liniger model and Yang-Mills theory on a twosphere, Phys. Rev. A 93, 013627 (2016).

[60] L. Piroli and P. Calabrese, Local correlations in the attractive one-dimensional Bose gas: From Bethe ansatz to the GrossPitaevskii equation, Phys. Rev. A 94, 053620 (2016).

[61] See Supplemental Material at http://link.aps.org/ supplemental/10.1103/PhysRevLett.123.160401, Appen$\operatorname{dix} \mathrm{A}$, for the specification of the truncated model (2), (3); Appendix B, for the derivation of the classical limit (6)-(8) of the model; Appendix C, for the derivation of universal separatrix quantization; Appendix D, for the separatrix quantization applied to the model, yielding (11); Appendix E, for the derivation of asymptotic spectral equidistance; Appendix F, for the derivation of (12) including finite-size corrections of order $\mathcal{O}(1)$ and details on the evolution of the one-body entropy; Appendix G, for a brief overview of our implementation of the extended five-mode model, which includes Refs. [62-70].

[62] Y. Castin, Simple theoretical tools for low dimension Bose gases, J. Phys. IV France 116, 89 (2004).

[63] M. Olshanii, Atomic Scattering in the Presence of an External Confinement and a Gas of Impenetrable Bosons, Phys. Rev. Lett. 81, 938 (1998).

[64] Y. Nishida, Universal bound states of one-dimensional bosons with two- and three-body attractions, Phys. Rev. A 97, 061603(R) (2018).

[65] L. Pricoupenko, Pure confinement-induced trimer in onedimensional atomic waveguides, Phys. Rev. A 97, 061604 (R) (2018).

[66] G. Guijarro, A. Pricoupenko, G. E. Astrakharchik, J. Boronat, and D. S. Petrov, One-dimensional three-boson problem with two- and three-body interactions, Phys. Rev. A 97, 061605 (R) (2018).

[67] C. Gardiner and P. Zoller, Quantum Noise: A Handbook of Markovian and Non-Markovian Quantum Stochastic Methods with Applications to Quantum Optics, Springer Series in Synergetics (Springer, Berlin, 2004).

[68] L. Pitaevskii and S. Stringari, Bose-Einstein Condensation and Superfluidity, International Series of Monographs on Physics (Oxford University Press, Oxford, 2016).

[69] R. M. Corless, G. H. Gonnet, D. E. G. Hare, D. J. Jeffrey, and D. E. Knuth, On the Lambert- $W$ function, Adv. Comput. Math. 5, 329 (1996).

[70] R. Mathew and E. Tiesinga, Phase-space mixing in dynamically unstable, integrable few-mode quantum systems, Phys. Rev. A 96, 013604 (2017).

[71] G. Dvali, D. Flassig, C. Gomez, A. Pritzel, and N. Wintergerst, Scrambling in the black hole portrait, Phys. Rev. D 88, 124041 (2013).
[72] G. Dvali and M. Panchenko, Black hole type quantum computing in critical Bose-Einstein systems, arXiv: 1507.08952.

[73] G. Dvali and C. Gomez, Black holes as critical point of quantum phase transition, Eur. Phys. J. C 74, 2752 (2014).

[74] C. S. Gerving, T. M. Hoang, B. J. Land, M. Anquez, C. D. Hamley, and M.S. Chapman, Non-equilibrium dynamics of an unstable quantum pendulum explored in a spin-1 Bose-Einstein condensate, Nat. Commun. 3, 1169 (2012).

[75] G. Arwas, A. Vardi, and D. Cohen, Superfluidity and Chaos in low dimensional circuits, Sci. Rep. 5, 13433 (2015).

[76] M. Prüfer, P. Kunkel, H. Strobel, S. Lannig, D. Linnemann, C.-M. Schmied, J. Berges, T. Gasenzer, and M. K. Oberthaler, Observation of universal dynamics in a spinor Bose gas far from equilibrium, Nature (London) 563, 217 (2018).

[77] M. A. Garcia-March, S. van Frank, M. Bonneau, J. Schmiedmayer, M. Lewenstein, and L. F. Santos, Relaxation, chaos, and thermalization in a three-mode model of a Bose-Einstein condensate, New J. Phys. 20, 113039 (2018).

[78] A. Kosior, A. Syrwid, and K. Sacha, Dynamical quantum phase transitions in systems with broken continuous time and space translation symmetries, Phys. Rev. A 98, 023612 (2018).

[79] B. M. Herbst and M. J. Ablowitz, Numerically Induced Chaos in the Nonlinear Schrödinger Equation, Phys. Rev. Lett. 62, 2065 (1989).

[80] M. Tabor, Chaos and Integrability in Nonlinear Dynamics: An Introduction (John Wiley and Sons, New York, 1989).

[81] A. M. O. de Almeida, Hamiltonian Systems: Chaos and Quantization (Cambridge University Press, Cambridge, England, 1990).

[82] T. Engl, J. Dujardin, A. Argüelles, P. Schlagheck, K. Richter, and J. D. Urbina, Coherent Backscattering in Fock Space: A Signature of Quantum Many-Body Interference in Interacting Bosonic Systems, Phys. Rev. Lett. 112, 140403 (2014).

[83] M. Albiez, R. Gati, J. Fölling, S. Hunsmann, M. Cristiani, and M. K. Oberthaler, Direct Observation of Tunneling and Nonlinear Self-Trapping in a Single Bosonic Josephson Junction, Phys. Rev. Lett. 95, 010402 (2005).

[84] R. Gati, M. Albiez, J. Fölling, B. Hemmerling, and M. K. Oberthaler, Realization of a single Josephson junction for Bose-Einstein condensates, Appl. Phys. B 82, 207 (2006).

[85] E. M. Graefe and H. J. Korsch, Semiclassical quantization of an N-particle Bose-Hubbard model, Phys. Rev. A 76, 032116 (2007).

[86] Q. Hummel, Semiclassical theory of few- and many-body quantum systems with short-range interactions, Ph.D. thesis, Universität Regensburg, 2018.

[87] We are not aware of renormalization group calculations of critical exponents in this model.

[88] G. Berman and G. Zaslavsky, Condition of stochasticity in quantum nonlinear systems, Physica (Amsterdam) 91A, 450 (1978).

[89] G. Zaslavsky, Hamiltonian Chaos and Fractional Dynamics (Oxford University Press, New York, 2005).

[90] B. Rauer, S. Erne, T. Schweigler, F. Cataldini, M. Tajik, and J. Schmiedmayer, Recurrences in an isolated quantum many-body system, Science 360, 307 (2018). 